\title{
Self-Efficacy Appraisals and Test-Taking Behavior of Students from Culturally Diverse Populations
}

\author{
Menucha Birenbaum and Fadia Nasser-Abu Alhija \\ School of Education, Tel Aviv University, Ramat Aviv, 69978 Tel Aviv, Israel \\ Correspondence should be addressed to Menucha Birenbaum; biren@post.tau.ac.il
}

Received 23 February 2013; Accepted 25 March 2013

Academic Editors: N. Dumais and B. Marlow

Copyright (C) 2013 M. Birenbaum and F. Nasser-Abu Alhija. This is an open access article distributed under the Creative Commons Attribution License, which permits unrestricted use, distribution, and reproduction in any medium, provided the original work is properly cited.

\begin{abstract}
This study examined the relationships between self-efficacy calibration and test-taking behavior as measured by the percentage of skipped items, unreached items, and incorrect responses on a mathematics test. Jewish and Arab 8th graders in four achievement levels, as defined by quartiles of the test score distribution, were compared with respect to their mathematics self-efficacy and their test-taking behavior. The results indicated that in every achievement level Arab students as compared to their Jewish counterparts tended to report higher levels of self-efficacy and to attempt more items on the test, which resulted in a higher rate of incorrect responses. The results support previous research findings pointing to the detrimental effect that overestimation of SE has on performance. The results were discussed with reference to the metacognitive processes involved in estimation of self-efficacy and in test performance. The identified gaps between the two ethnic groups were discussed in light of the learning culture that characterizes each of them. The contribution of the Israeli context, whereby students from two culturally diverse groups study according to the same mathematics curriculum but in separate schools, to understanding factors underlying culture-related group differences in mathematics test performance was underscored.
\end{abstract}

\section{Introduction}

Self-efficacy (SE) is considered a central mechanism of personal agency [1,2]. It refers to a person's capability to exercise some measures of control over his/her own functioning and over environmental events. More specifically, SE beliefs are context specific evaluations of a person's capability "to organize and execute the course of action required to produce given attainments" [1, page 2]. Such beliefs are claimed to affect thought patterns and performance on a wide variety of tasks. According to Bandura [1] SE beliefs are formed on the basis of four sources: past experience, vicarious experience (observation of others), social persuasion, and physiological state.

Empirical studies have lent support to the contention that SE plays a pivotal role in human functioning (e.g., [3-7]). In the area of education SE has been identified as a significant predictor of achievement in various subjects [8-10].

While a great deal of research has been devoted to examining the effect of SE on various aspects of learning, a paucity of research is evident regarding the accuracy of SE appraisals and the ways in which miscalibration of SE (i.e., incongruence between efficacy beliefs and actual performance) affects learning and test performance. Although it is claimed that moderately optimistic estimates of SE are instrumental to successfully completing challenging tasks because they foster perseverance and effort $[1,6,11]$, it has been shown that gross overestimation can have a detrimental effect on achievement. Thus, proficient performance necessitates resemblance between one's self-efficacy beliefs and his/her knowledge and skills [6].

The question addressed in the current study is whether SE calibration is related to cultural differences. The Israeli context, wherein students of two culturally diverse ethnic groups-Jews and Arabs-study according to the same mathematics curriculum but in separate schools, provides a suitable setting for such research. To address the research question, the current study focuses on the relationships between calibration of mathematics SE and test behavior of Jewish and Arab eighth graders in Israel. Prior to stating 
the rationale and explaining the design of the study, a brief description of the Israeli context is provided.

The Jewish and the Arab populations in the State of Israel represent two ethnic groups in a conflictual relationship with little intergroup contact [12]. The Arab minority constitutes approximately $21 \%$ of the population [13] and comprises four communities: Muslim, Christian Arabs, Druze, and Bedouin. The Arab minority is considered a nonassimilating minority and has limited access to the opportunity structure [14]. Consequently, the Arab minority has a lower standing in all aspects of socioeconomic status (including education, occupation, and income) as compared to the Jewish majority [14-16].

By contrast to the westernized Jewish mainstream community, the Israeli Arab community can be characterized as a developing society that tends to be conservative and patriarchal maintaining a clear and well-defined system of values and customs $[17,18]$.

Although officially all government schools in Israel are open to all students, in fact there are segregated educational systems for Arabs and Jews, both of which are run by the Ministry of Education. The languages of instruction in the Jewish and Arab school systems are Hebrew and Arabic, respectively. Yet, both systems have the same curricula in science and mathematics.

Previous studies of math achievement carried out on a national sample of 8th graders indicated a large achievement gap between the Jewish and Arab populations [19]. Following the publication of the 1996 National Assessment Test (NAT) results in mathematics, a more in-depth study, yet on a smaller scale, was designed in an attempt to account, at least partially, for the achievement gap. This was accomplished by examining the learning cultures in Jewish and Arab math classes, using a mixed method design [20]. This paper reports findings from part of the in-depth study, which examined one of the motivation factors, namely, students' SE, and its relations to their test-taking behavior.

Previous research has shown that estimation of SE involves self-awareness and task analysis, two skills that entail activation of metacognitive strategies (e.g., [21]). It can therefore be hypothesized that failing to use such strategies will lead, in addition to overestimation of SE, to an attempt to answer test items that are beyond one's capability, or achievement level, which is likely to result in erroneous responses on these items.

The literature regarding the paradigm shift in instruction that characterizes the "knowledge age" strongly suggests that learning environments based on constructivist principles promote the development of metacognitive skills [22-24]. In Israel, such environments are more common in the Jewish than in the Arab educational institutions $[14,25]$. In the current study it was therefore hypothesized that, on a given mathematics achievement level, Arab students on the average will report higher levels of mathematics SE than Jewish students; they will also attempt more items on a mathematics test which will result in a higher rate of incorrect responses.

\section{Method}

2.1. Participants. The research sample consisted of 476 eighth graders, 189 Jewish students ( 82 girls and 107 boys) and 287 Arab students (151 girls and 136 boys), from 12 middle schools (six Jewish and six Arab) located in the central district of Israel. The six Jewish and six Arab schools were selected to represent schools with low, medium, and high mathematics achievement levels and corresponding levels of social economic status in each community. The participating schools were selected on the basis of results of NAT-M, 1996, and the official SES index which officially was calculated for each school.

\subsection{Instruments}

2.2.1. SE Questionnaire. An eleven-item five-point Likerttype scale measuring students' beliefs in their capability to solve various types of mathematics tasks. The general format of the scale was adopted from Schwartzer [26] and the item content was tailored to eighth-grade mathematics. Responses ranged from 1 (strongly disagree) to 5 (strongly agree) and the mean score of the participant's responses was used as a measure of SE. The internal consistency coefficients (Cronbach's alphas) for the SE scale in the Jewish and Arab groups and in the entire sample, were $.80, .84$ and .83 , respectively.

2.2.2. Mathematics Test. Form A of the 1996 National Assessment Test in Mathematics (NAT-M) was delivered as part of the current study. The 1996 NAT-M was based on the formal curriculum issued by the Israeli Ministry of Education. The items on the test were developed by senior mathematics teachers and pedagogical consultants and were then translated into Arabic and reviewed by teachers and experts in mathematics education in that sector. All items were approved by the mathematics committee and were selected for inclusion in the operational version on the basis of their psychometric properties as identified in a pilot study [19]. Each version of the test consisted of three parts pertaining to twelve topics. About $25 \%$ of the items addressed topics studied in the 7 th grade. Topics that can be taught either in the 8th or in the 9th grade were addressed in a basic level only. The use of calculators was permitted in parts two and three of the test.

Form A of the test included 34 items, nine of which were of the choice response format (multiple choice or true-false with no negative marking for missed/incorrect answers) and the rest were of the constructed response format. One item was defined as "non-conventional" and one was a multistep investigation task (made up part three of the test). Since some of the items included more than one part, the total number of questions in Form A of the test was 44. The internal consistency coefficients (Cronbach's alphas) for Form A of the test in the Jewish and Arab groups and in the entire sample were $.90, .87$, and .91 , respectively.

2.2.3. Indicators of Test-Taking Behavior. The following percentages were computed for each student: percentage of 
missing responses (i.e., percentage of items to which the student did not provide an answer). This indicator was further broken into two components: percentage of skipped items (i.e., percent of missing responses before the last item answered by the student) and percentage of unreached items (i.e., percent of missing responses following the last item answered by the student). In addition, the percentages of correct and incorrect responses were computed.

2.2.4. Achievement Levels. Each student was classified into one of four achievement levels based on his/her total test score. The cutoff scores for the four levels were the 25th, 50th, and 75th percentiles of the total score distribution in the entire sample which corresponded to the following raw scores: $28.04,44.51$, and 60.23 , respectively. In other words, participants were grouped according to four achievement levels where Level 1 indicated the lowest achievement while Level 4 stood for the highest achievement.

\section{Results}

Mean comparisons between the Jewish and Arab groups on the SE scale and on the indicators of test-taking behavior were carried out using independent $t$-tests in the total sample as well as in every achievement level.

The results for the total sample indicated a significant difference between the two ethnic groups with respect to the SE score $(t=5.56, d=.54, P<.001)$, with Arab students scoring on the average higher than Jewish students $(M=$ $3.75, M=3.38$, resp.). Two other significant differences were detected with respect to percentages of correct and incorrect responses $(t=12.16, d=1.14, P<.001$, and $t=-19.67, d=$ $1.71, P<.001$, resp.). The Jewish group compared to the Arab group had a higher mean percentage of correct responses ( $M=57.47, M=36.51$, resp. $)$ and a lower mean percentage of incorrect response ( $M=20.89, M=42.28$, resp.). The discrepancies with respect to the other indicators of testtaking behavior, namely, percentage of missing, skipped, and unreached items, were insignificant.

The comparisons between the two groups in each of the achievement levels are presented in Table 1 . As it can be seen in the table, SE yielded significant discrepancies in three of the four achievement levels (2nd, 3rd, and 4th) with effect sizes of 1.04, 0.95, and 1.16, respectively. Arab students on the average reported higher levels of SE than did their Jewish counterparts. Discrepancies with respect to percentage of correct responses were insignificant in three achievement levels; only in the fourth achievement level the Jewish group outperformed the Arab group. The four discrepancies with respect to percentages of incorrect responses were significant with effect sizes ranging from 1.24 to 2.06. In every achievement level, Arab students on the average had a higher percent of incorrect responses than did their Jewish counterparts. The four discrepancies in percentage of missing responses as well as those for one of its components, namely, percentage of skipped items on the test, were significant with effect sizes ranging from .55 to 1.86. In every achievement level Jewish students on the average missed and skipped more items than did their Arab counterparts. On the other component of missing responses, namely, percentage of unreached items, significant discrepancies were detected in three of the four achievement levels (2nd, 3rd, and 4th) with effect sizes of $.87, .68$, and .51, respectively, again, with higher means in the Jewish group than in the Arab one.

\section{Discussion}

The results of the current study indicated that at any given achievement level, the Arab group compared to the Jewish group reported a higher level of SE along with a lower rate of missing responses, including both skipped and unreached items, and consequently had a higher rate of incorrect responses. These findings support the research hypotheses regarding differences between Jewish and Arab students with regard to SE calibration and test-taking behavior.

Metacognitive knowledge according to Brown [27] can be classified into three categories: declarative, procedural, and conditional. The first refers to self-awareness regarding one's skills, competencies, and abilities as a learner; the second refers to knowledge of how to use strategies for performing particular tasks; and the third refers to when and why to apply those strategies. Considering the effect of metacognitive knowledge on performance Flavell and Wellman [28] suggested three classification categories: knowledge of the person, of the task, and of the strategy. The first refers to the individual's beliefs regarding him/herself and others as cognizant creatures, the second refers to knowledge regarding the scope of various tasks and their requirements as well as to factors and conditions due to which some tasks are more difficult than others, and the third refers to knowledge regarding general and specific cognitive strategies and to the awareness of their usefulness for performing certain tasks.

Following these conceptualizations, it can be argued that the underlying pattern identified in the current study, of overestimating SE in combination with attempting items that are beyond one's achievement level, is a deficit in metacognitive knowledge regarding the task at hand, the person, or both. The former reflects a lack of understanding the task requirements, which results in a faulty task analysis, whereas the latter reflects an inaccurate appraisal of one's own capabilities to perform the task successfully, which may stem from low mathematics self-awareness. The question is why this pattern is more pronounced in the Arab group than in the Jewish one.

A qualitative analysis of learning interactions observed in several Arab and Jewish mathematics classes [25] indicated a higher dominance of the teacher during the lesson in the Arab classes. In general, Arab students were more passive compared to Jewish students, and rehearsal of rules and procedures was more pronounced in the Arab classes. In the Jewish classes, especially in the ones with high achievement, students were activated and encouraged to solve the problems autonomously before the teacher demonstrated the solution, to offer alternative solution routes, and to explain and justify their solution. Moreover, they were taught strategies of how to approach the problem, how to interpret and analyze it, and how to evaluate their solution. Such attributes of the instruction-learning process have been shown to promote 


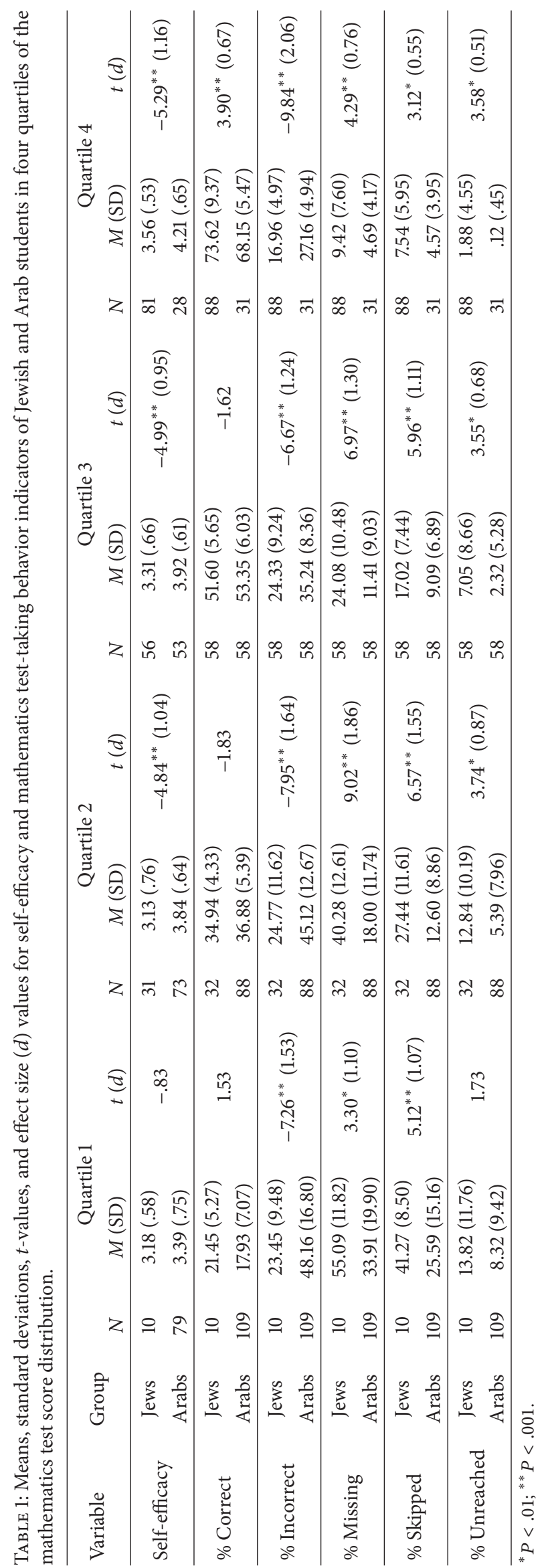


metacognitive skills as well as other self-regulated competencies $[29,30]$. The differences observed in the classroom practices may possibly account for part of the differences in SE and test-taking behavior between the students from the two cultural groups.

The findings of the current study seem to carry significant implications for instruction. Firstly, teachers need to be aware of their students' SE beliefs and of the accuracy of those appraisals. In case of gross overestimation they should design an intervention that, as suggested by Pajares [6, 11], would aim not at lowering the student's efficacy beliefs but rather at improving his/her calibration skills by promoting metacognitive knowledge and skills (i.e., self-awareness and task analysis), tailoring instruction to students' capabilities, and fostering the model of self-reflection [6]. This can be done by teaching strategies of task analysis and by increasing the learner's self-awareness through reflection on whether she/he has the resources to address the problem. Katz has demonstrated the effectiveness for improving SE calibration of combining repeated reflections on SE with strategy instruction, compared to any one of these components separately [31] Secondly, teachers have to promote their students' test-taking skills, one important component of which is metacognition. In a study about students' reported test-taking skills Roth and her associates [32] found that low achievers reported filling in answers on a standardized test without even reading the passage to which the questions were related. The authors refer to metacognition as one possible explanation. They point out those students' "lack of understanding about the appropriate conditions for using strategies, their poor causal reasoning about the connections between strategy use and test scores, and their poor judgments about the perceived utility of the strategy" (page 81). Thirdly, teachers should be responsive to the learning needs of students from diverse cultures and design differential intervention accordingly. For instance, in our study Arab students could benefit from a combined treatment aimed at improving their mathematics self-awareness and their test-taking strategies. Those in the lower achievement levels (69\% of the Arab sample), would additionally need remedial instruction for improving their mathematics skills and strategies. Compared to their Arab counterparts, Jewish students, especially those in the lower achievement levels (22\% of the Jewish sample), could benefit from a treatment addressing their deficiency in mathematics skills and strategies.

As for further studies, they should aim to carry out a more in-depth analysis of the processes involved in making appraisals of SE and in test-taking behavior and compare various cultural groups with respect to these processes. It should be noted that the current study examined SE appraisals and test-taking behavior using indirect measures. Moreover, the SE measure used in this study was not tailored to the task at hand but was rather a general measure addressing the type of tasks encountered in school mathematics. According to Bandura [1], the better the SE measure is tailored to the task at hand, the better predictor it is of performance on that particular task. Further research should therefore assess both SE and test-taking behavior using qualitative tools such as analysis of protocols taken while students are faced with the test, probing them about the strategies they use for appraising their SE as well as regarding their test-taking strategies.

On final note, contrary to findings of the current study, previous comparisons between minority and majority students with respect to SE resulted in lower means for the minority students [33]. The conflicting results between the current study and the other studies may be attributed to differences in the educational contexts. As already noted, Jews and Arabs in Israel study according to the same mathematics curriculum but in separate schools with almost no intergroup contact whereas most other research on ethnic group differences was carried out in integrated school systems [34]. It seems that the lack of contact between students of the two ethnic groups prevents Arab students from comparing their achievement level to that of the Jewish students. Such a comparison could have affected their self-belief system, particularly their self-efficacy, which is shaped, among other factors, by peer comparisons [1].

A rival hypothesis could attribute the gap in SE between Jewish and Arab students to cultural differences in the SE structure or in SE norms. As to the former, cross-cultural comparisons confirmed the robustness of the underlying structure [35]. As to the latter, although in cross-cultural comparisons mean differences were found to vary across nations [35], the fact that in the current study both factors, SE and test behavior, were found to vary in the direction hypothesized based on an underlying common denominator, namely, metacognitive skills, may be taken to refute the rival hypothesis. From a more general perspective the results of the current study can hence contribute to the accumulating knowledge regarding factors accounting for observed culture-related gaps in mathematics test performance.

\section{Acknowledgment}

The study reported in this paper was partially supported by a grant from the Israeli Ministry of Education. The ideas presented, the statements made, and the views expressed are the sole responsibility of the authors.

\section{References}

[1] A. Bandura, Self-Efficacy: The Exercise of Control, Freeman, New York, NY, USA, 1997.

[2] A. Bandura, "Social cognitive theory: an agentic perspective," Annual Review of Psychology, vol. 52, pp. 1-26, 2001.

[3] S. P. Becker and R. K. Gable, "The relationship of self-efficacy and GPA, Attendance, and college student retention," NERA Conference Proceedings, paper 26, 2009, http://digitalcommons.uconn.edu/nera_2009/26.

[4] G. Holden, "The relationship of self-efficacy appraisals to subsequent health related outcomes: a meta-analysis," Social Work in Health Care, vol. 16, no. 1, pp. 53-93, 1991.

[5] G. Holden, M. S. Moncher, S. P. Schinke, and K. M. Barker, "Self-efficacy of children and adolescents: a meta-analysis," Psychological Reports, vol. 66, no. 3 I, pp. 1044-1046, 1990.

[6] F. Pajares, "Self-efficacy during childhood and adolescence: implications for teachers and parents," in Self-Efficacy Beliefs 
of Adolescents, F. Pajares and T. C. Urdan, Eds., pp. 339-367, information Age Publishing, Charlotte, NC, USA, 2006.

[7] A. D. Stajkovic and F. Luthans, "Self-efficacy and work-related performance: a meta-analysis," Psychological Bulletin, vol. 124, no. 2, pp. 240-261, 1998.

[8] K. D. Multon, S. D. Brown, and R. W. Lent, "Relation of selfefficacy beliefs to academic outcomes: a meta-analytic investigation," Journal of Counseling Psychology, vol. 38, no. 1, pp. 3038, 1991.

[9] E. L. Usher, "Sources of middle school students? self-efficacy in mathematics: a qualitative investigation," The American Educational Research Journal, vol. 46, no. 1, pp. 275-314, 2009.

[10] B. J. Zimmerman, "Self-efficacy: an essential motive to learn," Contemporary Educational Psychology, vol. 25, no. 1, pp. 82-91, 2000.

[11] F. Pajares, "Self-efficacy beliefs in academic settings," Review of Educational Research, vol. 66, no. 4, pp. 543-578, 1996.

[12] V. Kraus, "The opportunity structure of young Israel Arabs," in Arab-Jewish Relations in Israel, J. E. Kraus and J. E. Hofman, Eds., Wymdham Hall, Bristol, UK, 1988.

[13] Central Bureau of statistics, Statistical Abstracts of Israel, no. 63, Central Bureau of statistics, Jerusalem, Israel, 2012.

[14] M. Al-Haj, Education, Empowerment and Control: The Case of the Arabs in Israel, State University of New York Press, Albany, NY, USA, 1995.

[15] V. Lavy, "Disparities between Arabs and Jews in school resources and student achievement in Israel," Economic Development and Cultural Change, vol. 47, no. 1, pp. 174-192, 1998.

[16] M. Semyonov and N. Lewin-Epstein, "Ethnic labor markets, gender and socioeconomic inequality: a study of Arabs in the Israeli labor force," Sociological Quarterly, vol. 35, no. 1, pp. 5168, 1994.

[17] Y. Batrice, The Palestinian Women in Israel: Reality and Challenges: An Empirical Study, Dar Alaswar, Ako, Israel, 2000.

[18] H. Sharabi, Introduction to Studying the Arab Population, Dar Alaswar, Ako, Israel, 1987.

[19] NAT-M (National Assessment Test-Mathematics for grade eight), Final Report, National Institute for Testing and Evaluation and The Ministry of Education, Jerusalem, Israel, 1996.

[20] J. W. Creswell, Educational Research: Planning, Conducting and Evaluating Quantitative and Qualitative Research, Prentice Hall, Upper-Saddle River, NJ, USA, 2002.

[21] A. Bandura and D. H. Schunk, "Cultivating competence, self-efficacy, and intrinsic interest through proximal selfmotivation," Journal of Personality and Social Psychology, vol. 41, no. 3, pp. 586-598, 1981.

[22] J. G. Brooks and M. G. Brooks, In Search of Understanding: The Case For Constructivist Classrooms, Association for Supervision and Curriculum Development, Alexandria, VA, USA, 1993.

[23] C. T. Fosnot, "Constructivism: a psychological theory of learning," in Constructivism: Theory, Perspectives, and Practice, C. T. Fosnot, Ed., Teachers College Press, New York, NY, USA, 1996.

[24] E. J. Langer, "A mindful education," Educational Psychologist, vol. 28, no. 1, pp. 43-50, 1993.

[25] M. Birenbaum and F. Nasser, "Mathematics achievement of Jewish and non-Jewish students and their relationships to students' and teachers' characteristics, and to the educational context," Final Report 0902, Tel Aviv University, School of Education, 2002.

[26] R. Schwartzer, Ed., Self-Efficacy: Thought Control of Action, Hemishere, Washington, DC, USA, 1992.
[27] A. L. Brown, "Knowing when, where and how to remember: a problem of metacognition," in Advances in Instructional Psychology, R. Glaser, Ed., vol. 1, pp. 77-165, Erlbaum, Hillsdale, NJ, USA, 1978.

[28] J. H. Flavell and H. M. Wellman, "Metamemory," in Perspectives on the Development of Memory and Cognition, R. V. Kail and J. W. Hagen, Eds., pp. 3-33, Erlbaum, Hillsdale, NJ, USA, 1977.

[29] J. Randi and L. Corno, "Teacher innovation in self-regulated learning," in Handbook of Self-Regulation, M. Boekaerts, P. R. Pintrich, and M. Zeidner, Eds., pp. 651-686, Academic Press, San Diego, Calif, USA, 2001.

[30] Z. R. Mevarech and B. Kramarski, "IMPROVE: a multidimensional method for teaching mathematics in heterogeneous classrooms," The American Educational Research Journal, vol. 34, no. 2, pp. 365-394, 1997.

[31] S. Katz, Effect of Reflection on Appraisal of Self-Efficacy in Regulating Audience Awareness in Writing Activities, Tel Aviv University, School of Education, 2001.

[32] J. L. Roth, S. G. Paris, and J. C. Turner, "Students' perceived utility and reported use of test-taking strategies," Issues in Education, vol. 16, no. 1/2, pp. 67-83, 2000.

[33] M. W. Mayo and N. Christenfeld, "Gender, race, and performance expectations of college students," Journal of Multicultural Counseling and Development, vol. 27, no. 2, pp. 93-101, 1999.

[34] W. F. Tate, "Race-ethnicity, SES, gender, and language proficiency trends in mathematics achievement: an update," Journal for Research in Mathematics Education, vol. 28, no. 6, pp. 652679, 1997.

[35] R. Schwarzer and A. Born, "Optimistic self-beliefs: assessment of general perceived self-efficacy in 13 cultures," World Psychology, vol. 3, no. 1-2, pp. 177-190, 1997. 

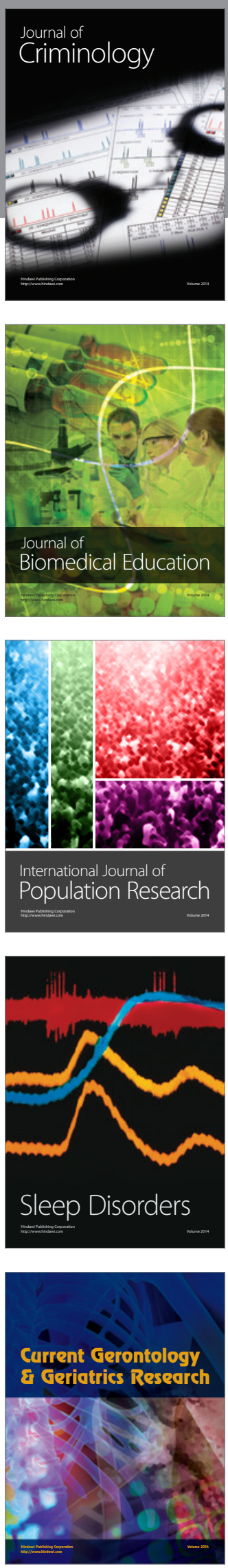
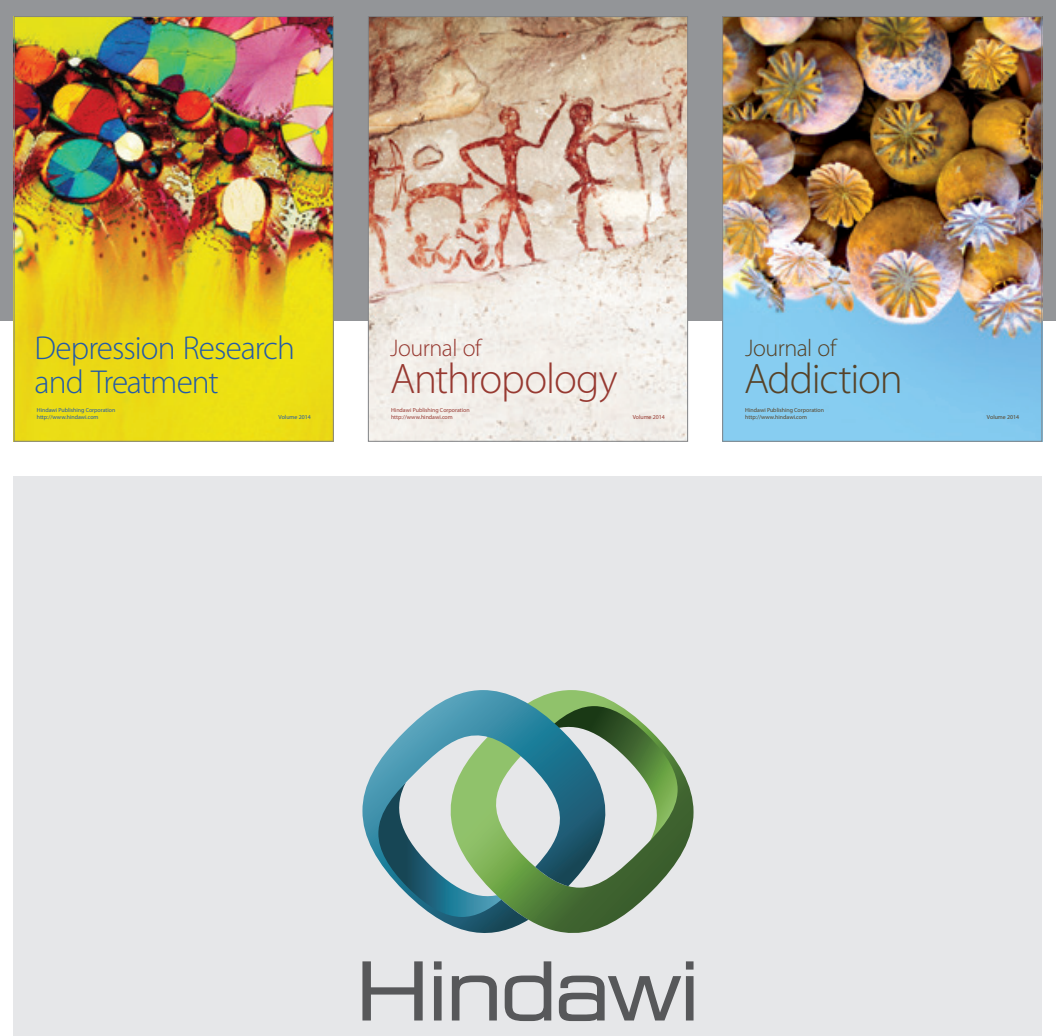

Submit your manuscripts at

http://www.hindawi.com

Child Development Research
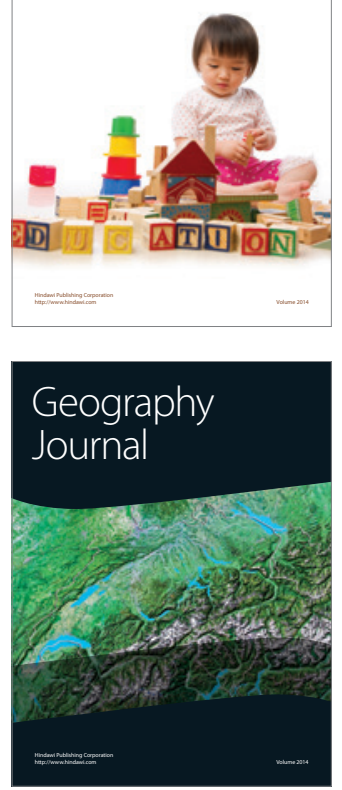

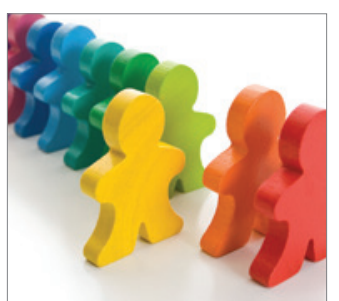

Autism

Research and Treatment
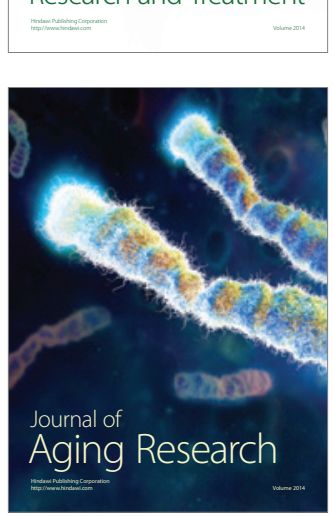
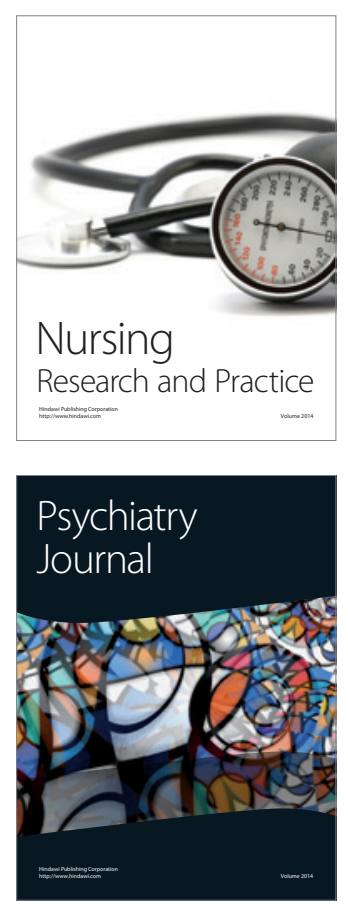
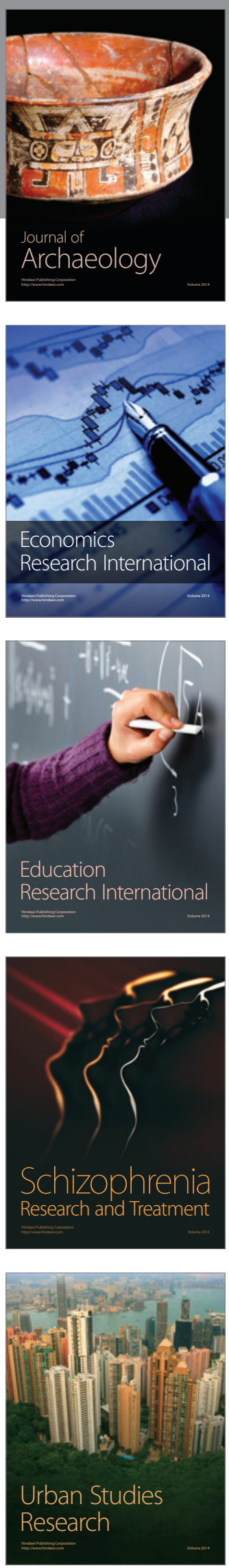